\section{Plantar vein thrombosis: a rare differential diagnosis in patients} with plantar pain

Tromboflebite plantar: um diagnóstico diferencial raro em pacientes com dor plantar

\section{Dear Editor}

A female, 42-year-old, smoking patient with previous history of thrombotic thrombocytopenic purpura, undergoing corticosteroid and plasmapheresis therapy was admitted to the hospital presenting with important pain in the plantar region of her right foot, with difficulty in ambulation starting about one week ago. The patient denied the occurrence of any trauma, previous surgery or recent travel. At physical examination, only hyperalgesia was observed at palpation. Magnetic resonance imaging (MRI) of the right foot demonstrated thickening and failure in filling in the whole extent of the lateral plantar vein associated with perivenular enhancement and edema of adjacent structures, suggesting the presence of plantar vein thrombosis (Figure 1).
Supplementary ultrasonography showed the presence of hypoechogenic material within de lateral plantar vein in association with ectasia and non-compressibility of the vessel, as well as absence of flow at Doppler study - findings which corroborated the initial hypothesis (Figure 2). The patient was discharged with recommendations for rest and treatment with nonsteroidal antiinflammatory drugs (NSAIDs), and presented with symptoms improvement at clinical follow-up.

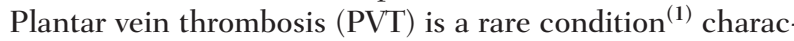
terized by development of an intraluminal thrombus in plantar veins, with less than 100 cases published in the literature ${ }^{(2)}$. It preferentially affects women of mean age 58.2 years $^{(3)}$. Generally, the lateral plantar vein is most affected (in $96 \%$ of cases), followed by the middle plantar vein $(\text { in } 41 \%)^{(3)}$. Several causal factors are speculated, among them traumas ${ }^{(4,5)}$, paraneoplastic syndromes $^{(6,7)}$, postoperative conditions ${ }^{(4,7)}$, thrombophilias ${ }^{(2,6)}$, use of contraceptive drugs ${ }^{(2,4)}$, immobilization ${ }^{(5,6)}$ and HIV infection ${ }^{(7)}$. However, most cases are classified as being idiopathic ${ }^{(2)}$.

Figure 1. A: Proton density-weighted sequence with fat saturation demonstrating parietal thickening of lateral plantar vein in association with perivascular edema (arrow). B,C: Contrast enhanced T1-weighted sequence showing intraluminal filling defect and perivascular enhancement (arrowheads).

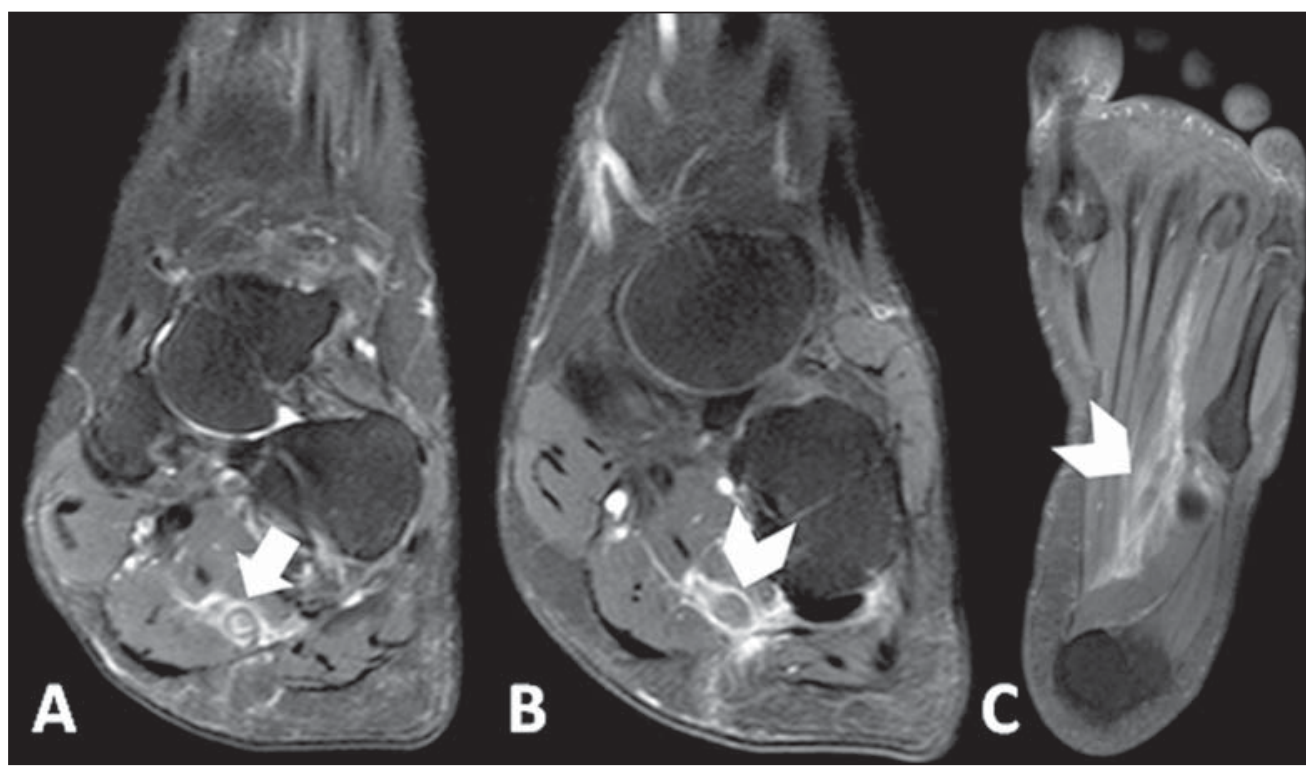

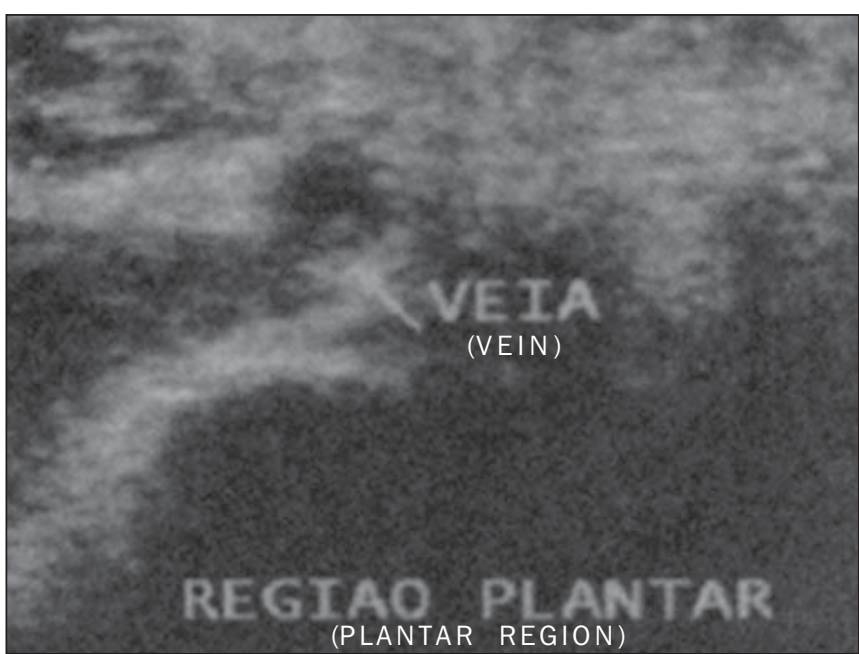

Figure 2. Ultrasonography of plantar region showing hypoechoic material within the lateral plantar vein in association with ectasia and non-compressibility of the vessel, as well as absence of flow at Doppler study.
Cardinal signs and symptoms of PVT include recent onset ${ }^{(7,8)}$ of plantar pain ${ }^{(1-3)}$ and local edema with a typically unilateral presentation $^{(1)}$ in association with limited ambulation ${ }^{(2)}$.

Considering the wide spectrum of differential diagnoses, the imaging diagnosis methods represent a useful tool for a correct characterization of the disease.

Ultrasonography is considered to be the main imaging method for the diagnosis of $\mathrm{PVT}^{(1,3)}$, demonstrating hypoechoic venous content ${ }^{(2,6)}$ associated with ectasia ${ }^{(2,5,6)}$, loss of vascular compressibility $^{(2,4)}$ and absence of flow at Doppler study ${ }^{(2,6)}$. However, plantar veins are neglected at routine examinations ${ }^{(1,2,4)}$, which may explain the low rate of diagnosis of the disease.

MRI has gained prominence as besides making the diagnosis of PVT it can rule out other possible causes of plantar pain ${ }^{(2)}$. Main findings include characterization of intraluminal filling defect in plantar veins ${ }^{(2,4,6)}$ in association with edema ${ }^{(5,6)}$ and perivenular enhancement ${ }^{(5)}$. Such findings were observed in $100 \%$ of cases in one of the greatest studies approaching the theme ${ }^{(5)}$. On the other hand, only in 2008 the first case of PVT diagnosed at MRI was described ${ }^{(9)}$. 
There is no defined treatment for PVT ${ }^{(1,3)}$, and possible treatment strategies include use of anticoagulant $\operatorname{drugs}^{(1-4,6)}$, NSAIDs $^{(1,3,6)}$, elastic socks ${ }^{(3,6)}$ and rest ${ }^{(6)}$. However, the different therapies have shown similar results.

The most important complications of PVT include thrombosis extension into deep veins in the $\mathrm{leg}^{(7)}$ and occurrence of pulmonary embolism ${ }^{(1)}$.

Amongst the differential diagnosis of PVT, plantar fasciitis ${ }^{(2,4,5)}$, tendinous involvement ${ }^{(3,5)}$, bursitis ${ }^{(5)}$, Morton's neuroma ${ }^{(4,5)}$, stress fractures $^{(2,4,5)}$, sesamoiditis ${ }^{(5)}$ and ganglion cysts ${ }^{(5)}$. No description of death associated with PVT is found in the literature.

\section{REFERENCES}

1. Barros M, Nascimento I, Barros T, et al. Plantar vein thrombosis and pulmonary embolism. Phlebology. 2015;30:66-9.

2. Bruetman JE, Andrews JA, Finn BC, et al. Plantar vein thrombosis as a cause of local pain. Medicina (B Aires). 2014;74:87-8.

3. Czihal M, Röling J, Rademacher A, et al. Clinical characteristics and course of plantar vein thrombosis: a series of 22 cases. Phlebology. 2014. [Epub ahead of print].
4. Karam L, Tabet G, Nakad J, et al. Spontaneous plantar vein thrombosis state of the art. Phlebology. 2013;28:432-7.

5. Miranda FC, Carneiro RD, Longo CH, et al. Tromboflebite plantar: achados em ressonância magnética. Rev Bras Ortop. 2012;47:765-9.

6. Geiger C, Rademacher A, Chappell D, et al. Plantar vein thrombosis due to busy night duty on intensive care unit. Clin Appl Thromb Hemost. $2011 ; 17: 232-4$.

7. Barros MV, Labropoulos N. Plantar vein thrombosis - evaluation by ultrasound and clinical outcome. Angiology. 2010;61:82-5.

8. Bernathova M, Bein E, Bendix N, et al. Sonographic diagnosis of plantar vein thrombosis: report of 3 cases. J Ultrasound Med. 2005;24:101-3.

9. Siegal DS, Wu JS, Brennan DD, et al. Plantar vein thrombosis: a rare cause of plantar foot pain. Skeletal Radiol. 2008;37:267-9.

Maurício Fabro ${ }^{1}$, Sara Raquel Madalosso Fabro ${ }^{1}$, Rafael Santiago Oliveira Sales ${ }^{1}$, Cesar Augusto Machado ${ }^{1}$, Gustavo Lopes de Araújo ${ }^{1}$

1. Hospital Santa Catarina de Blumenau, Blumenau, SC, Brazil. Mailing Address: Dr. Maurício Fabro. Rua Tobias Barreto, 266, ap. 304, Vila Nova. Blumenau, SC, Brazil, 89035-070. E-mail: mauriciofabro@hotmail.com.

http://dx.doi.org/10.1590/0100-3984.2015.0075

\section{Pulmonary neoplasia mimicking fungus ball}

Neoplasia pulmonar simulando bola fúngica

\section{Dear Editor,}

We report the case of a 74-year-old man smoking 80 cigarette packages per year, with history of pulmonary tuberculosis for 50 years. Two years ago, the patient underwent chest computed tomography that demonstrated centrilobular and paraseptal emphysema, besides sparse bullae, the largest one located in the right lower lobe, with a small nodular mass inside, measuring about $0.8 \mathrm{~cm}$ in diameter (Figure 1A).

The patient didn't return for follow-up and after two years presented with progressive dyspnea whose onset had occurred two months ago, in association with cough, weight loss and pain in the lower third of the right hemithorax. A new chest computed tomography demonstrated a mass with spiculated margins, adjacent to the posterior portion of the largest bulla, occupying the whole bulla where the nodular mass had been seen at the previous computed tomography images (Figure 1B). Also, interstitial thickening suggestive of carcinomatous lymphangitis was observed, besides bilateral pleural effusion.
Pericardium biopsy and cytological analysis of pleural effusion revealed adenocarcinoma, raising the hypothesis of lung adenocarcinoma with metastasis to the pleura and pericardium. A chemotherapy protocol with gemcitabine and carboplatin was initiated. The patient presented worsening of the respiratory condition, progressing to death after two months.

Lung cancer frequently presents like a nodule or solitary lung mass $^{(1,2)}$. However, the disease presentation forms are quite variable and some typical findings may be observed. One of such findings is growth from a preexisting cystic mass, mimicking a fungus ball. Thus, a cystic image showing either focal or diffuse wall thickening progressing to a nodular mass should include lung tumor in the differential diagnosis ${ }^{(3)}$, particularly in cases where the nodule is attached to the wall and does not move with change in decubitus.

Other conditions which may present the finding of fungus ball include Rasmussen aneurysms, hydatid cysts, abscesses and intracavitary hematomas, besides fungal diseases themselves (aspergillosis, nocardiasis, actinomycosis, candidiasis, coccidioidomycosis $)^{(2,4)}$.

As the neoplasm develops in previous pulmonary lesions, it is found especially in fibroatelectatic or granulomatous areas result-

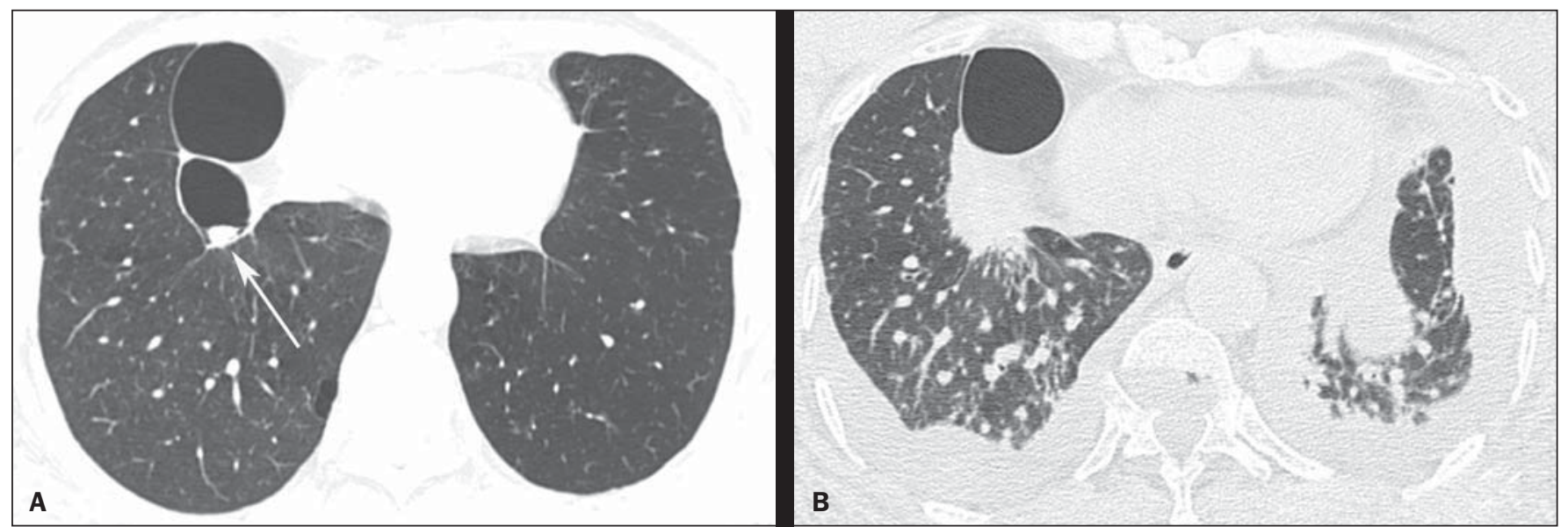

Figure 1. HRCT scan at the level of the lung bases (A) showing two bullae at right, with a small nodular mass measuring about $0.8 \mathrm{~cm}$ in diameter inside the small bulla (arrow). On B, scan acquired two years later, with a section of the same region, showing the presence of a mass with spiculated borders, adjacent to the posterior portion of the largest bulla, occupying the small bulla where the nodular mass had been seen at the previous CT images. Also, observe the presence of interstitial thickening suggestive of carcinomatous lymphangitis, besides bilateral pleural effusion. 